\title{
Resonant Cavity Enhanced Photodiodes in the Short-Wave Infrared for Spectroscopic Detection
}

\author{
Andrew Bainbridge, Katarina Mamic, Laura A. Hanks, Furat Al-Saymari, Adam P. Craig \\ and Andrew R. J Marshall
}

\begin{abstract}
The design, fabrication and characterization of resonant cavity enhanced photodiodes for the short-wave infrared has been investigated. An InGaAsSb absorber and AlGaSb barrier were used in an $n B n$ structure, within a Fabry-Perot cavity bounded by AlAsSb/GaSb DBR mirrors. The resonant cavity design produced a narrow response at $2.25 \mu \mathrm{m}$, with a FWHM of $\sim 26 \mathrm{~nm}$ and peak responsivity of $0.9 \mathrm{~A} / \mathrm{W}$. The photodiodes exhibited high specific detectivities and low leakage currents at $300 \mathrm{~K}-5 \times 10^{10} \mathrm{cmHz}^{1 / 2} \mathrm{~W}^{-1}$ and $0.2 \mathrm{mAcm}^{-2}$ respectively, with an applied bias voltage of $-100 \mathrm{mV}$. A maximum specific detectivity of $1 \times 10^{11} \mathrm{cmHz}^{1 / 2} \mathrm{~W}^{-1}$ was achieved at $275 \mathrm{~K}$ and the detector continued to perform well at high temperatures - at $350 \mathrm{~K}$ the peak specific detectivity was $3 \times 10^{9} \mathrm{cmHz}^{1 / 2} \mathrm{~W}^{-1}$. The narrow resonant response of these detectors make them suitable for spectroscopic sensing, demonstrated by measurements of glucose concentrations in water. Concentrations as low as $1 \%$ were discriminated, limited only by the associated electronic systems.
\end{abstract}

Index Terms-Remote sensing, Spectroscopy, Infrared detectors, Photodetectors

\section{INTRODUCTION}

$\mathbf{R}$ ESONANT cavity enhanced photodiodes (RCE PD) are a promising technology for non-invasive biomarker detection [1], especially in the short-wave infrared (SWIR) where absorption in skin is low [2]. There are numerous substances that have absorption peaks in this region which would be desirable to measure non-invasively, for example: glucose, lactate [3] and acetone [4]. For this purpose RCE PDs have properties that make them ideal candidates for future systems. They have an intrinsic narrow linewidth that offers a high spectral selectivity for focused targeting of specific substances; and as each device blocks the majority of the out-of-band spectrum, multiple detectors can be used with a single emitter to each detect different substances simultaneously, without additional interference.

Future biomarker detection systems will likely be used significantly in wearable technology [5]; where the possibilities of optical sensors have been shown by photoplethysmography sensors incorporation into many wearable electronic devices [6]. These sensors usually use reflection measurements of light up to $1 \mu \mathrm{m}$ in wavelength to detect blood volume variations [7]; however, for biomarker detection it is beneficial to extend this wavelength range - for greater tissue penetration [8] and to be able to target specific absorption peaks. Higher spectral discrimination is also required. To be incorporated into wearable systems a device must be small and ideally with low power consumption [9], both of which RCE PDs can satisfy. The narrow linewidth removes the need for filters, reducing total device size, and they can be operated at room temperature

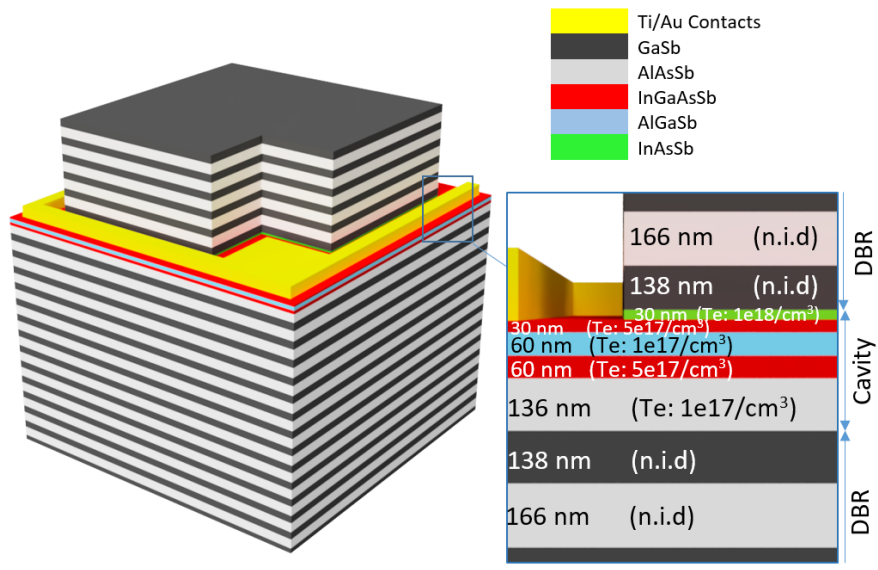

Fig. 1: The structure of the RCE PD. The AlAsSb and GaSb DBR mirrors enclose the cavity, which is made up of an AlAsSb buffer layer, InGaAsSb absorber layer, AlGaSb barrier layer, InGaAsSb contact layer and an InAsSb etch stop layer.

and above - without the cooling systems commonly required by infrared detectors [10].

The aim of this work is to demonstrate RCE PDs in the SWIR using GaSb based III-V materials and an $n B n$ structure, building upon previous work in the mid-wave infrared [11], [12]. In RCE PDs, high quality mirrors enhance the optical field intensity in the cavity and hence high absorption can be achieved in a significantly reduced absorber volume [13], when the absorber is placed at the anti-node in the field. The thinner absorber reduces Auger dark current mechanisms, which depend linearly on absorber volume [14] and if the absorber is fully depleted then generation-recombination mechanisms will be similarly reduced. The use of distributed Bragg reflector (DBR) mirrors, where reflectivity can be controlled by the number of repeat periods [15], affords control over spectral specificity and narrow line widths when desired.

RCE PDs are shown to offer the ideal combination for spectroscopic sensing of high quantum efficiency, low leakage current and high spectral specificity. The sample device has a resonant peak close to an absorption peak of glucose, at $\sim 2.2 \mu \mathrm{m}$, which has been used to test the principles of sensing biomarkers with RCE PDs.

\section{DESIGN AND FABRICATION}

The RCE PD structure - shown in figure 1 - uses two $\mathrm{AlAsSb} / \mathrm{GaSb}$ DBRs either side of the cavity and an $n B n$ structure in the cavity. The cavity layer structure was adapted from previous research on $\mathrm{InGaAsSb} n B n$ detectors [16], using 


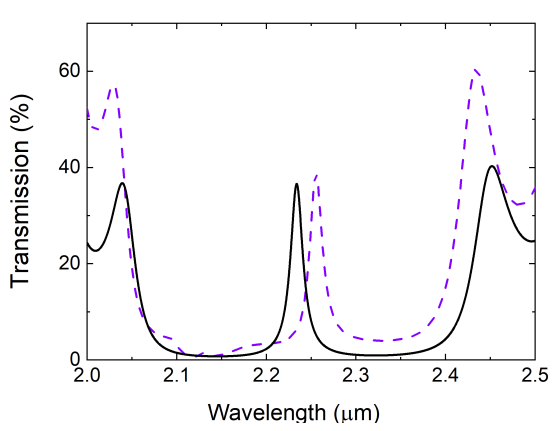

(a)

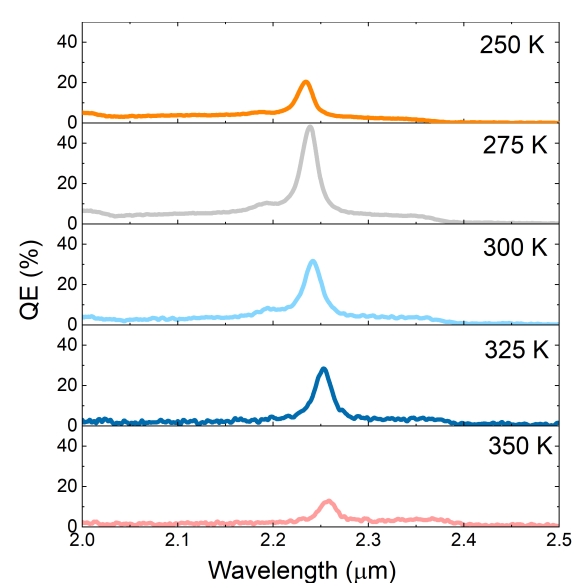

(b)

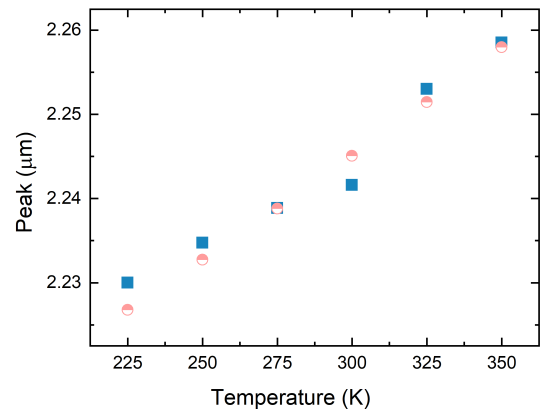

(c)

Fig. 2: (a) Transmission through the unprocessed sample (solid line), measured by FTIR, compared to a transmission line model of the device structure (dashed line). (b) Temperature dependent QE - in $25 \mathrm{~K}$ increments. (c) Temperature dependence of the peak wavelength - measured (squares) and modelled (circles).

a similar InGaAsSb absorber and AlGaSb barrier materials, but with the absorber volume decreased by more than a factor of 30 - to take advantage of the resonant enhancement factor. Layer thicknesses of: $60 \mathrm{~nm}$ absorber, $60 \mathrm{~nm}$ barrier and 30 $\mathrm{nm}$ contact layer were used. A cavity spacer layer $(136 \mathrm{~nm}$ thick) is included beneath the absorber - to place the absorber at the centre of the cavity, at the peak of intensity of the optical field.

The sample was grown by molecular beam epitaxy on a tellurium-doped, n-type (001) GaSb substrate. A $500 \mathrm{~nm}$ thick $\mathrm{GaSb}$ buffer layer was followed by the layers shown in figure 1 The bottom DBR consisted of 13 repeats of the two layers, followed by the five cavity layers, then the top DBR - of one layer of $\mathrm{GaSb}$ then five repeats of $\mathrm{AlAsSb} / \mathrm{GaSb}$. Growths rates were approximately $1 \mathrm{ML} / \mathrm{s}$ for all layers.

$\mathrm{X}$-ray diffraction analysis of the alloys in the reported device showed lattice matching to within 300 arcsecs for the $\mathrm{AlAs}_{0.08} \mathrm{Sb}, \mathrm{Al}_{0.36} \mathrm{GaSb}$ and $\mathrm{In}_{0.24} \mathrm{GaAs}_{0.21} \mathrm{Sb}$ alloys (hereafter 'AlAsSb', 'AlGaSb' and 'InGaAsSb' respectively). The thin $\mathrm{InAs}_{0.80} \mathrm{Sb}$ (hereafter 'InAsSb') etch-stop layer exhibited some mismatch; however, it was outside of the electrically active region of the structure.

The top mirror and InAsSb etch-stop layer were etched outside the device area and the top contact was placed on top of the top InGaAsSb layer - so current would not be extracted through the DBRs - and the device mesa was then defined by etching the top InGaAsSb layer. Standard photolithography, wet etchants and Ti/Au thermal evaporation were used throughout.

\section{RESUlts}

Figure $2 \mathrm{a}$ shows the transmission of the RCE PD at room temperature - measured by FTIR. The absorption peak - at $2.25 \mu \mathrm{m}$ - sits very close to the centre of the stop-band $(2.23 \mu \mathrm{m})$, this indicates that the cavity is only slightly thicker than is ideal - a $20 \mathrm{~nm}$ reduction in thickness would match the DBRs perfectly. Also shown is an ideal model curve for the transmission. The transmission line model [13] used incorporates the refractive indices, absorption coefficients and thicknesses of each layer to determine the total impedance of the structure; then used to calculate the transmission. The absorption coefficient of the absorber was experimentally determined to be $7000 \mathrm{~cm}^{-1}$ at $2.25 \mu \mathrm{m}$; all other layers were assumed to have absorption coefficients of zero at this wavelength. Literature values were used for the refractive indices of all materials [17]. The modelled transmission shows an ideal device, with the absorption peak in the centre of the stop-band.

The resonant peak wavelength is determined simply by the layer thicknesses, limited by the cutoff wavelength of the InGaAsSb absorber. With this material system it would be straightforward to create a device with a resonant response up to the maximum cutoff wavelength; $2.8 \mu \mathrm{m}$ has been shown to be possible [18]. The lower level broadband response either side of the resonant peak is due to the post-growth fabrication of the sample and is not intrinsic to the structure. A portion of the absorber between the perimeters of the two mesas on each device extends beyond the top DBR - see figure 1 - and is exposed to the full spectrum of incident light. This nonresonant response could be removed by covering the exposed areas of the absorber.

Room temperature quantum efficiency $(\mathrm{QE})$ measurements were taken using a Bentham PVE300 quantum efficiency system, which overfills the RCE PD area with a constant flux. A PbS reference detector was used to calculate the $\mathrm{QE}$ of the device. Temperature dependent $\mathrm{QE}$, figure $2 \mathrm{~b}$, was calculated using FTIR spectral response measurements, calibrated by the room temperature $\mathrm{QE}$ measurement. The $\mathrm{QE}$ increases with temperature up to a maximum of $48 \%$ at $275 \mathrm{~K}$ and decreases thereafter, with a $300 \mathrm{~K} \mathrm{QE}$ of $33 \%$. This initial increase and then decrease with temperature could be due to a transition from a radiative dominated minority carrier lifetime to either Shockley-Read-Hall or Auger dominated [19]. Without the resonant enhancement an absorber of this thickness would 


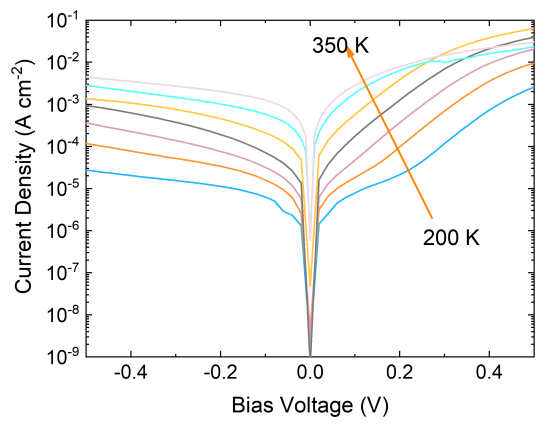

(a)

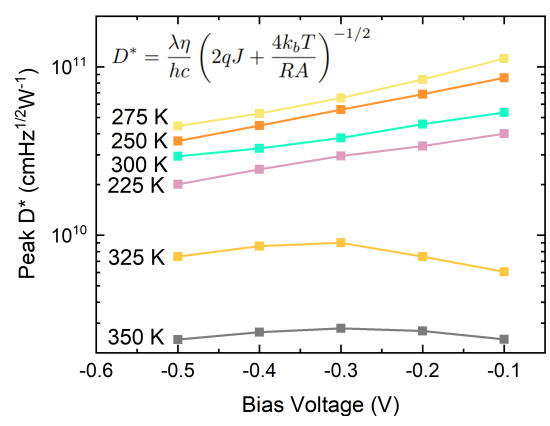

(b)

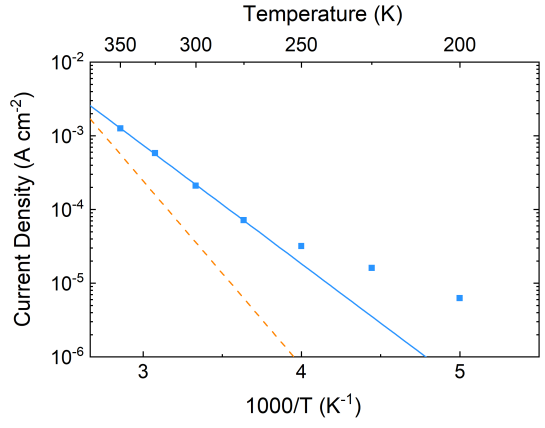

(c)

Fig. 3: (a) Temperature dependent leakage currents (25 K increments). (b) Temperature and voltage dependence of peak specific detectivity. (c) Arrhenius plot comparison of the RCE PD (squares) all measured with an applied negative bias voltage of 100 $\mathrm{mV}$. Activation energy of $319 \mathrm{meV}$ (solid line) is shown. Rule 07 for a $2.4 \mu \mathrm{m}$ cutoff wavelength (dashed line) is included for comparison.

have a maximum possible $\mathrm{QE}$ of $4 \%$; in other words the enhancement effect of the cavity increases the QE by up to a factor of 12 .

The resonant wavelength of the detector shifts with temperature, from $2229 \mathrm{~nm}$ to $2258 \mathrm{~nm}$ between $225 \mathrm{~K}$ and $350 \mathrm{~K}$. This is an approximately linear change that equates to 0.23 $\mathrm{nm} / \mathrm{K}$ - shown in figure $2 \mathrm{c}$, this is significantly lower than the temperature dependence of cutoff wavelength of the InGaAsSb absorber, which is $1.4 \mathrm{~nm} / \mathrm{K}$. The main source of the shift with temperature is changes in refractive indices of the layers, determined by the Moss relation [20]. The calculated shift in peak wavelength - plotted in figure $2 \mathrm{c}$ - closely match the measured values, confirming that this is the dominant cause of the shift. The shift in peak wavelength due to the thermal expansion of the layers was also calculated, and found to be approximately 25 times smaller. The FWHM of the of the resonant peak was determined by a Gaussian fit to each peak and is approximately $26 \mathrm{~nm}$. There is a slight increase with temperature, about $2 \mathrm{~nm}$ over the temperature range $250 \mathrm{~K}$ to $350 \mathrm{~K}$. This FWHM gives a Q factor of approximately 90, comparable to the best reported Q factors for RCE PDs [12], [21], with the highest value reported to be 95 .

Leakage currents were characterized in a cryogenic probestation with an integrated cold shield - shown in figure 3a Device area dependence of the dark current density was calculated to be approximately constant with area, so perimeter currents were negligible, as expected for the $n B n$ architecture [22]. Compared to similar, but thicker $\mathrm{nBn}$ structures the RCE PDs have approximately 10 times lower leakage currents at $300 \mathrm{~K}$ [16], [18]. An Arrhenius plot for the RCE PD and the reference detector is shown in figure 3c The activation energy for the RCE PD with an applied bias voltage of $-100 \mathrm{mV}$ as used for the QE measurements in figure $2 b$ - is shown to be $319 \mathrm{meV}$. This is lower than the bandgap of the absorber - $\sim 500 \mathrm{meV}$ - indicating that the Fermi level is not at the band edge, possibly due to strong band bending in the very thin absorber, resulting in the presence of Shockley-Read Hall currents. Rule 07 [23], a bandgap dependent metric for stateof-the-art $\mathrm{HgCdTe}$ detectors is also shown - this metric is for standard thickness MCT devices, which III-V detectors of similar thickness are usually unable to match. However, at and above room temperature the leakage current of the RCE PD is close to the Rule 07 line, indicating the enhancement attributed to the thin absorber used in the resonant cavity design.

Peak specific detectivity was calculated taking into consideration both Johnson and shot noise - figure $3 \mathrm{~b}$ At $300 \mathrm{~K}$ the RCE PD showed a peak specific detectivity of $5 \times 10^{10} \mathrm{cmHz}^{1 / 2} \mathrm{~W}^{-1}$, with the maximum value of $1 \times 10^{11} \mathrm{cmHz}^{1 / 2} \mathrm{~W}^{-1}$ achieved at $275 \mathrm{~K}$, with an applied bias voltage of $-100 \mathrm{mV}$. The specific detectivity of the RCE PD is maintained even at high temperatures, significant for allowing uncooled operation of the RCE PD, even at high ambient temperatures.

One of the potential uses for RCE PDs is spectroscopic sensing, with this material system being well suited to the detection of biomarkers including glucose, lactate and urea [24], which have absorption features in the SWIR. A first proof of concept of glucose detection has been made using two RCE PDs - with peaks at separate wavelengths of $2.13 \mu \mathrm{m}$ and $2.25 \mu \mathrm{m}$ - used to measure changes in glucose concentration in water. Multiple detectors are necessary to allow fluctuations unrelated to the glucose concentration to be monitored; for example, signal changes due to small fluctuations in temperature and source intensity can be identified and excluded this way. Figure 4 shows that the detector at $2.13 \mu \mathrm{m}$ detects the maximum change in transmission from a glucose solution in this wavelength range. However, there is some variation of water transmission with temperature at that particular wavelength. The detector at $2.25 \mu \mathrm{m}$ resolves a minimal change in transmission due to the presence of glucose and thus can be used to monitor transmission changes due to temperature and exclude the effects of fluctuations in system throughput. The transmission through glucose solutions were measured using a ratio of the output of the two detectors. The lowest concentration measured was $1000 \mathrm{mg} / \mathrm{dL}$ (1\%), limited as a result of electrical noise from the amplifiers in the simple proof of concept set-up. For comparison, concentrations of glucose in blood are $\sim 100-200 \mathrm{mg} / \mathrm{dL}$ [25]. With optimisation and refinement of the associated electronics, RCE PD's could benchmark non-invasive biosensing in this wavelength range. 

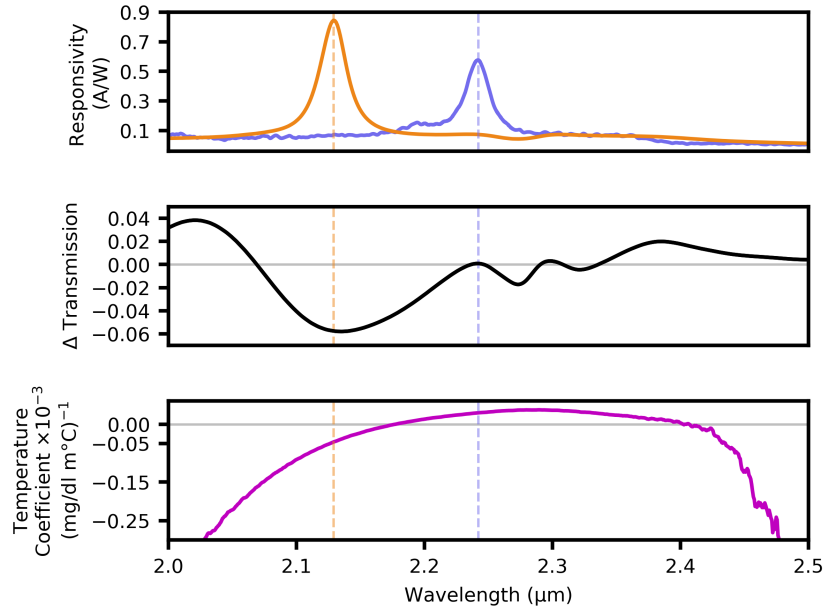

Fig. 4: Top: The responsivity of two RC photodetectors focused on $2.13 \mu \mathrm{m}$ (shown in orange) and $2.25 \mu \mathrm{m}$ (shown in blue) Dashed lines indicate the wavelength corresponding to the peak responsivity for each detector. Middle: The change in transmission between water and a glucose solution of $33 \%$ concentration taken at $31^{\circ} \mathrm{C}$. Bottom: The temperature coefficient of water, indicating the significance temperature has on transmission.

\section{SUMMARY}

In summary, a high performance resonant cavity-enhanced photodiode was demonstrated to exceed the performance of standard $n B n$ detectors using the same material system. A room temperature quantum efficiency of $33 \%$ was achieved with just a $60 \mathrm{~nm}$ absorber thickness, along with a peak specific detectivity of $5 \times 10^{10} \mathrm{cmHz}^{1 / 2} \mathrm{~W}^{-1}$. The RCE PD offers usable performance even at high ambient temperatures without cooling.

It would be straightforward to create RCE PDs with peak responses between $2.0 \mu \mathrm{m}$ and $2.8 \mu \mathrm{m}$ using this material system to target specific substances. The peak wavelength of the presented detector $-2.25 \mu \mathrm{m}$ - aligns to an absorption peak for glucose, allowing measurements of glucose concentrations in water down to $1 \%$ glucose.

\section{ACKNOWLEDGMENT}

The authors thank the EPSRC for the studenship of A. Bainbridge (EP/R513076/1), funding from Innovate UK (105014/22714) and DSTL (DSTLX:1000116341).

\section{REFERENCES}

[1] C. Balas, "Review of biomedical optical imaging-a powerful, noninvasive, non-ionizing technology for improving in-vivo diagnosis," Measurement Science and Technology, vol. 20, no. 10, p. 104020, sep 2009. [Online]. Available: https://doi.org/10.1088\%2F0957-0233\% 2F $20 \% 2 \mathrm{~F} 10 \% 2 \mathrm{~F} 104020$

[2] E. Hemmer, F. Vetrone, and K. Soga, "Lanthanide-based nanostructures for optical bioimaging: Small particles with large promise," MRS Bull., vol. 39, no. 11, pp. 960-964, 2014.

[3] V. SapTari and K. Youcef-Toumi, "Design of a mechanical-tunable filter spectrometer for noinvasive glucose measurement," Appl. Opt., vol. 43, no. 13, pp. 2680-2688, 2004.
[4] R. Peale, A. Muravjov, C. Fredricksen, G. Boreman, H. Saxnea, G. Braunstein, V. Vaks, A. Maslovsky, and S. Nikifirov, "Spectral signatures of acetone vapor from ultraviolet to millimeter wavelengths," Int. J. High Speed Electron. Syst., vol. 18, no. 3, pp. 627-637, 2008.

[5] J. Kim, A. Campbell, B. E.-F. de Ávila, and J. Wang, "Wearable biosensors for healthcare monitoring," Nat Biotechnol, vol. 37, p. 389-406, 2019.

[6] T. Tamura, Y. Maeda, M. Sekine, and M. Yoshida, "Wearable photoplethysmographic sensors-past and present," Electronics, vol. 3, no. 3, pp. 282-302, 2014.

[7] L. Lindberg and P. Oberg, "Photoplethysmography. part 2. influence of light source wavelength," Med. Biol. Eng. Comput., vol. 29, no. 1, p. 48-54, 1991.

[8] D. Naczynski, C. Sun, S. Türkcan, C. Jenkins, A. Koh, D. Ikeda, G. Pratx, and L. Xing, "X-ray-induced shortwave infrared biomedical imaging using rare-earth nanoprobes," Nano Lett., vol. 15, no. 1, pp. 96-102, 2015.

[9] J. Heikenfeld, A. Jajack, J. Rogers, P. Gutruf, L. Tian, T. Pan, R. Li, M. Khine, J. Kim, J. Wang, and J. Kim, "Wearable sensors: modalities, challenges, and prospects," Lab Chip, vol. 18, pp. 217-248, 2018. [Online]. Available: http://dx.doi.org/10.1039/C7LC00914C

[10] A. Stiff-Roberts, "Quantum-dot infrared photodetectors: a review," $J$. Nanophotonics, vol. 3, no. 1, p. 48-54, 2009.

[11] A. P. Craig, F. Al-Saymari, M. Jain, A. Bainbridge, G. R. Savich, T. Golding, A. Krier, G. W. Wicks, and A. R. Marshall, "Resonant cavity enhanced photodiodes on gasb for the mid-wave infrared," Applied Physics Letters, vol. 114, no. 15, p. 151107, 2019.

[12] V. Letka, A. Bainbridge, A. P. Craig, F. Al-Saymari, and A. R. J. Marshall, "Resonant cavity-enhanced photodetector incorporating a type-ii superlattice to extend mwir sensitivity," Opt. Express, vol. 27, no. 17, pp. 23970-23 980, 2019.

[13] M. Unlu and S. Strite, "Resonant cavity enhanced photonic devices," $J$. Appl. Phys., vol. 78, no. 2, pp. 607-639, 1995.

[14] J. Pautrat, E. Hadji, J. Bleuse, and N. Magnea, "Resonant-cavity infrared optoelectronic devices," J. Electron. Mater., vol. 26, no. 6, p. 667-672, 1997.

[15] C. Sheppard, "Approximate calculation of the reflection coefficient from a stratified medium," Pure Appl. Opt., vol. 4, no. 5, pp. 665-669, 1995.

[16] A. P. Craig, M. Jain, G. Wicks, T. Golding, K. Hossain, K. McEwan, C. Howle, B. Percy, and A. R. J. Marshall, "Short-wave infrared barriode detectors using ingaassb absorption material lattice matched to gasb," Appl. Phys. Lett., vol. 106, no. 20, p. 201103, 2015.

[17] S. Adachi, "Optical dispersion relations for gap, gaas, gasb, inp, inas, insb, alxga1xas, and in1xgaxasyp1y," Journal of Applied Physics, vol. 66, no. 12, pp. 6030-6040, 1989.

[18] H. Hao, G. Wang, X. Han, D. Jiang, Y. Sun, C.Guo, W. Xiang, Y. Xu, and Z. Niu), "Extended-wavelength ingaassb infrared unipolar barrier detectors," AIP Adv., vol. 8, no. 9, p. 095106, 2018.

[19] D. Donetsky, S. Anikeev, N. Gu, G. Belenky, S. Luryi, C. A. Wang, D. A. Shiau, M. Dashiell, J. Beausang, and G. Nichols, "Analysis of recombination processes in $0.5-0.6 \mathrm{ev}$ epitaxial gainassb lattice-matched to gasb," AIP Conference Proceedings, vol. 738, no. 1, pp. 320-328, 2004.

[20] S. K.Tripathy, "Refractive indices of semiconductors from energy gaps," Opt. Mater., vol. 46, pp. 240-246, 2015.

[21] C. L. Canedy, W. W. Bewley, C. D. Merritt, C. S. Kim, M. Kim, M. V. Warren, E. M. Jackson, J. A. Nolde, C. A. Affouda, E. H. Aifer, I. Vurgaftman, and J. R. Meyer, "Resonant-cavity infrared detector with five-quantum-well absorber and $34 \%$ external quantum efficiency

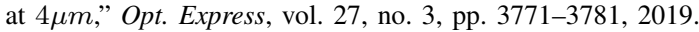

[22] J. Pedrazzani, S. Maimon, and G. Wicks, "Use of $n B n$ structures to suppress surface leakage currents in unpassivated inas infrared photodetectors," Electronics Letters, vol. 44, pp. 1487-1488(1), December 2008.

[23] W. Tennant, D. Lee, M. Zandian, E. Piquette, and M. Carmody, "Mbe hgcdte technology: A very general solution to ir detection, described by "rule 07", a very convenient heuristic," J. Electron. Mater., vol. 37, no. 9, p. 1406-1410, 2008.

[24] X. Liu, J. Liao, H. Song, Y. Yang, C. Guan, and Z. Zhang, "A biocharbased route for environmentally friendly controlled release of nitrogen: Urea-loaded biochar and bentonite composite," Sci. Rep., vol. 9, no. 9548, 2019

[25] T. Dall, W. Yang, P. Halder, B. Pang, M. Massoudi, N. Wintfeld, A. Semilla, J. Franz, and P. Hogan, "The economic burden of elevated blood glucose levels in 2012: Diagnosed and undiagnosed diabetes, gestational diabetes mellitus, and prediabetes," Diabetes Care, vol. 37, no. 12, p. 3172-3179, 2014. 